\title{
DIFFERENTIAL EQUATIONS INVARIANT UNDER FINITE REFLECTION GROUPS
}

BY

ROBERT STEINBERG

1. Introduction and statements of results. In this paper we study the characteristic functions (eigenfunctions) of those differential operators with constant coefficients that are invariant under finite linear groups, especially under finite reflection groups. Such operators and functions occur naturally in connection with harmonic analysis on semisimple Lie groups (see, e.g., [4]). Our main result (1.3 below) is a manifold of characterizations of finite reflection groups in terms of the above-mentioned characteristic functions.

To state this result, we introduce some notations which will be used throughout the paper. $V$ is a vector space of finite dimension $n$ over $K$, the complex field, $V^{*}$ is the dual of $V, S$ is the symmetric algebra on $V$, and $S^{*}$ is the algebra of entire functions on $V$. For $v$ in $V, D_{v}$ is the operator on $S^{*}$ defined by $\left(D_{v} F\right)(w)$ $=\lim t^{-1}(F(w+t v)-F(w))\left(t \in K, t \rightarrow 0 ; w \in S ; F \in S^{*}\right)$, and for $s$ in $S, D_{s}$ is then defined so as to make $D$ an isomorphism. The space $S^{*}$ (and also the space of polynomial functions on $V$ ) is in algebraic duality with $S$ via the inner product $(s, F)=\left(D_{s} F\right)(0)\left(s \in S, F \in S^{*}\right)$. Any automorphism $\sigma$ of $V$ acts directly on $S$ and contravariantly on $S^{*}$ by the rule $(\sigma F)(v)=F\left(\sigma^{-1} v\right)$; one has $\sigma\left(D_{s} F\right)=D_{\sigma s} \sigma F$, so that the above inner product is preserved. Whenever a group $G$ of automorphisms of $V$ is being considered, $I$ consists of the elements of $S$ invariant under $G, I_{0}$ consists of the elements of $I$ that vanish at 0 , and $S_{0}$ is the ideal in $S$ generated by $I_{0}$.

Assume now that $G$, of order $g$, is a finite group of automorphisms of $V$ and that $F$ is a nonzero element of $S^{*}$ which is a characteristic function of all of the operators $D_{i}(i \in I): D_{i} F=c_{i} F$. Then the map $i \rightarrow c_{i}$ is a homomorphism of the algebra $I$ into $K$, which can be extended [5, p. 420] to a homomorphism of $S$ into $K$; thus $c_{i}=i(L)$ for some $L$ in $V^{*}$ and all $i$ in $I$. We are thus led to consider, for each $L$ in $V^{*}$, the following system of differential equations, to be solved for $F$ in $S^{*}$.
$1.1\left(\Sigma_{L}\right)$
$D_{i} F=i(L) F \quad(i \in I)$.

We write $\Sigma_{L}$ for this system and $d_{L}$ for the dimension of the space of solutions of $\Sigma_{L}$. As a backdrop for our main theorem, we have the following preliminary results, of which at least $\left(b^{\prime}\right),(c),(d)$, and (e) are well known. Here,

Presented to the Society, February 22, 1962; received by the editors April 26, 1963. 
and elsewhere, $\Sigma_{0}\left(G_{L}\right)$ denotes the system defined in terms of the subgroup $G_{L}$ just as $\Sigma_{0}$ is defined in terms of $G$.

1.2 THEOREM. Let $G$ be a finite group of automorphisms of $V$, and for each $L$ in $V^{*}$ let $G_{L}$, of order $g_{L}$ be the subgroup that fixes $L$. (a) If $F_{0}$ is a solution of $\Sigma_{0}\left(G_{L}\right)$, then $F_{0} \exp L$ is a solution of $\Sigma_{L}$. (b) Each solution of $\Sigma_{L}$ can be written $F=\Sigma_{\sigma \in G} \sigma\left(P_{\sigma} \exp L\right)$, each $P_{\sigma}$ being a polynomial of degree at most $\left(g_{L}-1\right)^{n}$. (b') Each solution of $\Sigma_{0}$ is a polynomial of degree at most $(g-1)^{n}$. (c) The solutions of $\Sigma_{0}$ form the orthogonal complement of $S_{0}$ in $S^{*}$. Thus $d_{0}=\operatorname{dim} S / S_{0}$. (d) $g \leqq d_{L}<\infty$. (e) If $m$ is the number of elements in a minimal generating set for $I_{0}$, then $n \leqq m<\infty$.

This brings us to our main theorem. We define a reflection to be an automorphism of $V$ other than the identity which fixes a hyperplane pointwise and is of finite order (not necessarily two), and a reflection group to be a group generated by reflections.

1.3 THEOREM. If $G$ is a finite group of automorphisms of $V$, the following conditions are equivalent. (a) $G$ is a reflection group. (b) There exists a polynomial $P$ such that $s$ is in $S_{0}$ if and only if $D_{s} P=0$. (c) There exists a polynomial $P$ such that $F$ is a solution of $\Sigma_{0}$ if and only if $F=D_{s} P$ for some $s$ in $S$. (c') There exists a character $\varepsilon$ from $G$ into the multiplicative group of $K$ such that, if $P^{(L)}$ denotes a polynomial of minimal degree among those which transform under $G_{L}$ according to $\varepsilon$ and are nonzero, and if $P_{L}=g^{-1} \Sigma_{\sigma \in G} \varepsilon(\sigma)^{-1} \sigma\left(P^{(L)} \exp L\right)$, then $F$ is a solution of $\Sigma_{0}$ if and only if $F=D_{s} P_{L}$ for some s in $S$. (c') For each $L$ in $V^{*}$ there exists a polynomial $P^{(L)}$ such that $F_{0}$ is a solution of $\Sigma_{0}\left(G_{L}\right)$ if and only if $F_{0}=D_{s} P^{(L)}$ for some $s$ in $S$. (d) $d_{L}$ is independent of $L$. (e) $d_{0}=g$. $\left(\mathrm{e}^{\prime}\right) \operatorname{dim} S / S_{0}=g$. (f) If $i_{0}, i_{1}, \cdots, i_{r}$ in $I$ are such that $i_{0}$ is not in the ideal in $I$ generated by the others, and if $p_{0}, p_{1}, \cdots, p_{r}$ in $S$ are homogeneous and such that $\Sigma p_{a} i_{a}=0$, then $p_{0}$ is in $S_{0}$. (g) $I_{0}$ is generated, as an algebra over $K$, by $n$ of its elements.

That every finite reflection group has properties ( $\left.\mathrm{e}^{\prime}\right),(\mathrm{f})$ and $(\mathrm{g})$ is due to Chevalley [1] (he considers only reflections of order two, but his methods apply equally well to reflections of any order), and that (g)implies(a) is due to Shephard and Todd [7]. To amplify 1.3 we introduce some more notation. $H$ is the set of hyperplanes in which reflections of $G$ take place, and for each $h$ in $H, R_{h}$ is a nonzero element of $V^{*}$ which vanishes on $h$ and $e(h)$ is the order of the (necessarily cyclic) group generated by the reflections in $h$ that are in $G$.

1.4 THEOREM. Let $G$ be a finite reflection group on $V$. Let $\Pi$ denote the product of all $R_{h}^{e(h)-1}$ as $h$ varies over the reflecting hyperplanes for $G$, and for each $L$ in $V^{*}$ let $\Pi^{(L)}$ denote the corresponding product for the subgroup $G_{L}$ that fixes $L$, and let $\Pi_{L}=g^{-1} \Sigma_{\sigma \in G}\left(\operatorname{det} \sigma^{-1}\right) \sigma\left(\Pi^{(L)} \exp L\right)$. (a) If $P$ is a homogeneous 
polynomial for which 1.3(c) holds, then $P$ is a constant multiple of $\Pi$. (b) If $\varepsilon$ is such that $1.3\left(\mathrm{c}^{\prime}\right)$ holds, then $\varepsilon=$ det. (c) If $P^{(L)}$ and $P_{L}$ are such that $1.3\left(\mathrm{c}^{\prime}\right)$ holds, they are constant multiples of $\Pi^{(L)}$ and $\Pi_{L}$, respectively. (d) To within multiplication by a constant, $\Pi_{L}$ is the unique function which is a solution of $\Sigma_{L}$ and transforms according to det.

We remark that in case $V$ is a Cartan subalgebra of a semisimple Lie algebra $A$ over the complex field and $G$ is the corresponding Weyl group the polynomial $\Pi$ in 1.4 is just a constant multiple of the product of the positive roots (relative to some ordering of $V$ ) (see [4] where significant use is made of this polynomial), and, if $L$ is identified with an element of $V$, then the value at $L$ of any finite-dimensional irreducible character of $A$ is just the ratio of two appropriately chosen values of $\Pi_{L}$ (this is Weyl's formula; see, e.g., [6, pp. 255, 257] for the two most important cases: in the first, $L$ is not on any reflecting hyperplane, while in the second, $L$ is 0 ).

Clearly not every subgroup of a reflection group is a reflection group, but as a biproduct of 1.3 we prove:

1.5 THeOREM. Let $G$ be a reflection group on the space $V$ and let $U$ be a subspace of $V$. Then the subgroup of $G$ that fixes $U$ pointwise is also a reflection group.

From this we have the following corollaries, important in the context of Lie algebras (see $[6$, p. 242] or [8, pp. 16-106]).

1.6 COROLLARY. If $v$ is a vector not on any of the reflecting hyperplanes of a reflection group $G$, the only element of $G$ that fixes $v$ is the identity.

1.7 Corollary. Let $G$ be a reflection group, and assume that the linear functions $R_{h}$, defined just prior to 1.4 above, are such that for some vector $v$ every $R_{h}(v)$ has a positive real part. Then the only element of $G$ which permutes the $R_{h}$ among themselves is the identity.

As is well known, the solutions of a system of differential equations such as $\Sigma_{L}$ enjoy certain mean value properties, in other words, satisfy certain difference equations [5, pp. 435-439]. The connection will be discussed in $\$ 8$ below. Finally, to close this introduction, let us consider an example.

1.8 ExAMPLE. Let $x_{1}, x_{2}, \cdots, x_{n}$ be coordinates for $V$ and let $G$ be the symmetric group of degree $n$ acting via permutations of the coordinates. Then $G$ is generated by reflections in the hyperplancs $x_{i}-x_{j}=0(i<j)$, so that properties (b) and (c) of 1.3 hold with $P=\prod_{i<j}\left(x_{i}-x_{j}\right)$. This special case is due to E. Fisher and J. Schur [2], whose methods, however, are not applicable to the general case. That $1.3\left(\mathrm{e}^{\prime}\right)$ also holds here is a very old result. If we modify the example so that $G$ consists of permutations of the coordinates ccmbined with multiplications by arbitrary $r$ th roots of 1 , then $P$ is to be replaced by $\prod\left(x_{i}^{r}-x_{j}^{r}\right)$. 
The interested reader should have no trouble in constructing in these cases, for typical values of $L$, the function $\Pi_{L}$ of 1.4 as well as the differential equations $\Sigma_{L}$ and the corresponding difference equations $\Sigma_{L}^{\prime}$ considered in $\S 8$. For further examples, we refer to [7] where the classification of finite reflection groups is completed.

2. General properties of $\Sigma_{L}$. This section is devoted to the proof of 1.2. In the notation of (a), and with $i$ in $I$, we have $D_{i}\left(F_{0} \exp L\right)=(\exp L) D_{j} F_{0}$, with $j$ in $S$ defined by $j\left(L^{\prime}\right)=i\left(L^{\prime}+L\right)\left(L^{\prime} \in V^{*}\right)$. Here $j$ need not be invariant under $G$, but it is invariant under $G_{L}$. Thus $D_{j} F_{0}=j(0) F_{0}=i(L) F_{0}$, and (a) is proved.

2.1 LEMMA. If $F$ is a solution of $\Sigma_{L}$, it is also a solution of the system $\Gamma_{L}: D_{s} F=0\left(s=\prod_{\sigma \in G}\left(v-\left(\sigma^{-1} L\right)(v)\right), v \in V\right)$.

We have an identity $0=\prod_{\sigma \in G}(v-\sigma v)=v^{g}+i v^{g-1}+\cdots$ with $i, \cdots$ in $I_{0}$. Thus $D_{s} F=\left(D_{v}^{g}+i(L) D_{v}^{g-1}+\cdots\right) F$, which, by $\Sigma_{L}$, is equal to $\left(D_{v}^{g}+D_{i} D_{v}^{g-1}+\cdots\right) F$, which is equal to $D_{0} F$, that is, to 0 , by the identity above.

We now prove 1.2(b). By allowing $v$ in the lemma to vary over a basis of $V$, we see that each solution of $\Sigma_{L}$ can be written as the sum of a finite number of terms $P \exp L^{\prime}$, with $L^{\prime}$ a linear function and $P$ a polynomial. Consider a nonzero term of this form. Since $P \exp L^{\prime}$ is a solution of $\Gamma_{L}, P$ satisfies $D_{t} P=0$ with $t=\prod\left(v-\left(\sigma^{-1} L\right)(v)+L^{\prime}(v)\right)$. If $r$ (which depends on $v$ ) is the number of $\sigma$ in $G$ such that $\left(\sigma^{-1} L\right)(v)=L^{\prime}(v)$, we may write $D_{t} P=0$ as $D_{v}^{r} P=\left(c_{1} D_{v}+c_{2} D_{v}^{2}+\cdots\right)$ $D_{v}^{r} P\left(c_{j} \in K\right)$, whence, since $D_{v}^{r} P$ is a polynomial, $D_{v}^{r} P=0$. Now $L^{\prime}$ must equal some $\sigma^{-1} L$ since otherwise we could find $v$ so that $r$ is 0 and then conclude $P=0$. Consider the case $L^{\prime}=L$. We choose a basis $B$ for $V$ such that for $v$ in $B$ and $\sigma$ in $G$ we have $\left(\sigma^{-1} L\right)(v)=L(v)$ only if $\sigma$ is in $G_{L}$. For each $v$ in $B$, the number $r$ above is then $g_{L}$, so that the equations $D_{v}^{r} P=0$ imply that the degree of $P$ is at most $\left(g_{L}-1\right)^{n}$. Thus we have (b), and as a special case, also $\left(\mathrm{b}^{\prime}\right)$.

For (c), we have the chain of equivalent statements: $F$ is in the orthogonal complement of $S_{0} ;(s i, F)=0$ for all $s$ in $S$ and $i$ in $I_{0} ; D_{i} F=0$ for all $i$ in $I_{0} ; F$ is a solution of $\Sigma_{0}$.

Let $s_{1}, s_{2}, \cdots, s_{h}$ be homogeneous elements of $S$ which project into a basis for $S / S_{0}$. By induction on the degree, every element of $S$ can be put in the form $\sum i_{a} s_{a}$ with $i_{a}$ in $I$. By Galois theory [9, p. 156, Fundamental Theorem, part 4] applied to the quotient fields of $S$ and $I$, we get $h \geqq g$, which, by (c), is the same as $d_{0} \geqq g$. Writing $d_{0 L} \geqq g_{L}$ for the corresponding inequality for the group $G_{L}$, we have, by (a), $d_{L} \geqq d_{0 L} g / g_{L} \geqq g_{L} g / g_{L}=g$. By (b), we have $d_{L}<\infty$, hence (d).

Finally, $n \leqq m$ in (e) because the two quotient fields mentioned above have the same degree of transcendence over $K$, namely $n$, and $m<\infty$ easily follows from $\operatorname{dim} S / S_{0}<\infty$.

3. Proof of Theorem 1.3, first part. In this section we prove first that 1.3(a) implies $1.3(b)$ and that for $P$ we may take the polynomial $\Pi$ defined in 1.4 , and 
then we prove that $1.3(\mathrm{~b})$ implies $1.3(\mathrm{c})$. For convenience, we call a function $F$ skew if $\sigma F=(\operatorname{det} \sigma) F$ for every $\sigma$ in $G$.

3.1 Lemma. (a) The polynomial $\Pi$ is skew. (b) $\Pi$ divides every skew polynomial.

Proof. If $\sigma$ is a reflection in $G$ and $h$ is the corresponding hyperplane, then $\sigma R_{h}=\left(\operatorname{det} \sigma^{-1}\right) R_{h}$ and $(\operatorname{det} \sigma)^{e(h)}=1$. Thus $\sigma\left(R_{h}^{e(h)-1}\right)=(\operatorname{det} \sigma) R_{h}^{e(h)-1}$. If $k$ is a reflecting hyperplane different from $h$, there is a smallest integer $m$ such that $\sigma^{m} R_{k}=c R_{k}$ for some $c$ in $K$. Since $\sigma^{m} R_{k}-R_{k}$ is a multiple of $R_{h}$, we have $c=1$. Thus $\sigma$ preserves the product of $R_{k}, \sigma R_{k}, \cdots, \sigma^{m-1} R_{k}$, whence the $R_{l}^{e(l)-1}$ for which $l \neq h$ may be arranged in cycles so that $\sigma$ preserves the product of the terms in each cycle. Thus $\sigma \Pi=(\operatorname{det} \sigma) \Pi$, and since the reflections generate $G, \Pi$ is skew. Now, assume that $h$ and $\sigma$ are as above and also that $\sigma$ has order $e(h)$. Choose a basis $X, Y, \cdots$ of $V^{*}$ so that $X=R_{h}, \sigma Y=Y, \sigma Z=Z, \cdots$. Then $\sigma$ acts on a monomial $X^{j} Y^{k} \ldots$ via multiplication by $(\operatorname{det} \sigma)^{-j}$, a number which is equal to $\operatorname{det} \sigma$ only if $e(h)$ divides $j+1$, hence only if $j$ is at least $e(h)-1$. Thus every skew polynomial is divisible by $X^{e(h)-1}=R_{h}^{e(h)-1}$, and so also by $\Pi$.

Assuming now that $G$ is a finite reflection group, we prove 1.3(b) with $P=\Pi$. If $s$ is in $I_{0}, D_{s} \Pi$ is skew and of lower degree than $\Pi$; thus it is 0 by 3.1(b), and the "only if" part of 1.3(b) holds. Conversely, let $s$ in $S$ be homogeneous and such that $D_{s} \Pi=0$. We prove by downward induction on the degree of $s$ that $s$ is in $S_{0}$, the result being true for a sufficiently high degree because $\operatorname{dim} S / S_{0}$ is finite, by $1.2(\mathrm{c}, \mathrm{d})$. Let $\sigma$ be a reflection in $G$. We may choose $v$ in $V$ so that $\sigma v=(\operatorname{det} \sigma) v$. Then $t=v s$ has higher degree than $s$ and $D_{t} \Pi=0$. Thus by the induction hypothesis $v s$ is in $S_{0}$ and there is a relation $v s=\Sigma u_{p} i_{p}$ with $u_{p}$ in $S$ and $i_{p}$ in $I_{0}$. On applying $\sigma$ and then combining the two relations, we get $s-(\operatorname{det} \sigma) \sigma s=\Sigma\left(\left(u_{p}-\sigma u_{p}\right) / v\right) i_{p}$, an element of $S_{0}$. Because $G$ is a reflection group, we get $s \equiv(\operatorname{det} \sigma) \sigma s \bmod S_{0}$ for all $\sigma$ in $G$, and then by averaging over $G, s \equiv s^{\prime} \bmod S_{0}$, the element $s^{\prime}$ being such that $\sigma s^{\prime}=\left(\operatorname{det} \sigma^{-1}\right) s^{\prime}$. Applying 3.1 to the group $G^{*}$ dual to $G$, we may write $s^{\prime}=\pi i$, with $\pi$ defined for $G^{*}$ just as $\Pi$ is for $G$, and with $i$ in $I$. If $i$ has positive degree, $s^{\prime}$ is in $S_{0}$ and hence so is $s$. Assume then that $i$ is constant, that $Q$ is any homogeneous polynomial of the same degree as $\Pi$, and that $R$ is the average of $\left(\operatorname{det} \sigma^{-1}\right) \sigma Q$ under $G$. Then $R$ is a multiple of $\Pi$ by $3.1(\mathrm{~b})$, so that $D_{s^{\prime}} R=0$ and $\left(s^{\prime}, R\right)=0$. Thus $\left(s^{\prime}, Q\right)=g^{-1} \Sigma \sigma\left(s^{\prime}, Q\right)=g^{-1} \Sigma\left(\left(\operatorname{det} \sigma^{-1}\right) s^{\prime}, \sigma Q\right)=\left(s^{\prime}, R\right)=0$, and since $Q$ is arbitrary, $s^{\prime}=0$, so that $s$ is in $S_{0}$ in this case also, and 1.3(b) is proved.

3.2 Corollary. If $G$ is a reflection group and $\Pi$ is of degree $N$, then $S_{0}$ contains every homogeneous element of $S$ of degree greater than $N$, and $N$ is the smallest integer with this property.

Now we deduce 1.3(c) from 1.3(b). Let $D P$ denote the space of derivatives $D_{t} P$ of $P$. We have the chain of equivalent statements: $s$ is in the orthogonal 
complement of $D P ;\left(s, D_{t} P\right)=0$ for all $t$ in $S ;\left(t, D_{s} P\right)=0$ for all $t$ in $S ; D_{s} P=0$; $s$ is in $S_{0}$. The last equivalence is by the assumption 1.3(b). Thus $S_{0}$ is the orthogonal complement of $D P$. Because $D P$ is finite dimensional, it in turn is the orthogonal complement of $S_{0}$. Hence, by $1.2(\mathrm{c})$, it coincides with the space of solutions of $\Sigma_{0}$, which is $1.3(\mathrm{c})$.

4. Continuation of the proof. In this section we prove the equivalence of the conditions $(c),\left(c^{\prime}\right)$ and $\left(c^{\prime \prime}\right)$ in 1.3.

4.1 Lemma. (a) If $P$ satisfies 1.3(c), then $P_{0}$, the homogeneous part of highest degree of $P$, also does. (b) If $P$ is homogeneous and satisfies 1.3(c), there exists a character $\varepsilon$ on $G$ such that $\sigma P=\varepsilon(\sigma) P$ for all $\sigma$ in $G$. (c) If $P$ and $\varepsilon$ are as in (b) and $Q$ in $S^{*}$ satisfies $\sigma Q=\varepsilon(\sigma) Q(\sigma \in G)$, then $Q=c P+$ higher terms $(c \in K)$.

Proof. $P_{0}$ is a solution of $\Sigma_{0}$, whence $P_{0}=D_{s} P(s \in S)$, by $1.3(\mathrm{c})$, and clearly $s(0) \neq 0$. Hence the map $D_{t} P \rightarrow D_{s}\left(D_{t} P\right)=D_{t} P_{0}$ is an isomorphism of the space of solutions of $\Sigma_{0}$ into itself, and since the space is finite dimensional, the isomorphism is onto. Thus $P=D_{t} P_{0}$ for some $t$ in $S$, and (a) follows. If $P$ is as in (b), then $\sigma P$ satisfies $\Sigma_{0}$, so that $\sigma P=D_{s} P$ for some $s$ in $S$. Since $\sigma P$ and $P$ have the same degree, $s$ may be taken constant, $s=\varepsilon(\sigma)$, and clearly $\varepsilon$ is a character on $G$. In proving(c), we need only consider the case in which $Q$ is a nonzero homogeneous polynomial which transforms according to $\varepsilon$ and has minimal degree relative to these properties. Since for $i$ in $\mathrm{I}_{0}, D_{i} Q$ has smaller degree than $Q$, it must be 0 , so that $Q$ is a solution of $\Sigma_{0}$, and $Q=D_{s} P$ for some $s$ in $S$, by $1.3\left(\right.$ c). For $\sigma$ in $G, Q=D_{\sigma s} P$, so that by averaging over $G$ we may take $s$ in $I$. But then, because $P$ satisfies $\Sigma_{0}, Q=s(0) P$, which implies (c).

4.2 Lemma. Let $F$ satisfy $\Sigma_{L}$, and let $F=F_{r}+F_{r+1}+\cdots$ with $F_{j}$ a homogeneous polynomial of degree $j$. Then (a) $F_{r}$ satisfies $\Sigma_{0}$, and (b) if $P$ satisfies 1.3(c) and $r$ exceeds the degree of $P$, then $F=0$.

Proof. If $i$ is homogeneous and in $I_{0}$, all terms of degree less than $r$ in $D_{i} F=i(L) F$ must vanish, whence $D_{i} F_{r}=0$, which is (a). Thus by 1.3 (c) we have $F_{r}=D_{s} P(s \in S)$ in (b). Hence $F_{r}$ either vanishes or has degree at most that of $P$, which implies (b).

We come now to the proof that $1.3(\mathrm{c})$ implies $1.3\left(\mathrm{c}^{\prime}\right)$. For this we assume that $P$ is homogeneous (see 4.1(a)), that $\varepsilon$ is defined in terms of $P$ as in 4.1(b), and that $P^{(L)}$ and $P_{L}$ are defined in terms of $\varepsilon$ according to $1.3\left(\mathrm{c}^{\prime}\right)$. If $i$ is in $S$, homogeneous, of positive degree, and invariant under $G_{L}$, then $D_{i} P^{(L)}$ has smaller degree than $P^{(L)}$, hence is 0 because of the definition of $P^{(L)}$, whence $P^{(L)}$ is a solution of $\Sigma_{0}\left(G_{L}\right)$. By 1.2(a), then $P_{L}$ and all of its derivatives are solutions of $\Sigma_{L}$. We prove the converse in a sharpened form.

4.3 Lemma. If $s_{1}, s_{2}, \cdots, s_{h}$ are homogeneous elements of $S$ which project into a vector space basis in $S / S_{0}$, and if $P_{L}$ is as above, then every solution of $\Sigma_{L}$ has the form $F=D_{s} P_{L}$ with $s=\Sigma c_{j} s_{j}\left(c_{j} \in K\right)$. 
We prove this by downward induction on the number $r$ that occurs in any representation of $F$ in the form of 4.2, the result being true if $r$ is large enough by 4.2(b). By 4.2(a) and 1.3(c) we may write $F_{r}=D_{t} P$ with $t$ in $S$, and because $P$ is homogeneous and is annihilated by $D_{s}$ for every $s$ in $S_{0}$, we may take $t$ in the form $\sum a_{j} s_{j}\left(a_{j} \in K\right)$. Now $P_{L} \neq 0$ because distinct exponentials are linearly independent over the polynomials; so by 4.1 (c) and 4.2(b) we may write $P_{L}=c P+$ higher terms with $c \neq 0, c$ in $K$. But then $F-c^{-1} D_{t} P_{L}$ may be written in the form of 4.2 with $r+1$ in place of $r$, whence the induction hypothesis may be applied to complete the proof of 4.3 , and hence also the proof of $1.3\left(\mathrm{c}^{\prime}\right)$.

Assume now that $1.3\left(\mathrm{c}^{\prime}\right)$ holds. Because of the defining properties of $P^{(L)}$, we have already noted that $P^{(L)}$ is a solution of $\Sigma_{0}\left(G_{L}\right)$. Let $F_{0}$ be any solution of this system. By $1.2(\mathrm{a})$ and $1.3\left(\mathrm{c}^{\prime}\right)$, we may write $F_{0} \exp L=D_{t}\left(P^{(L)} \exp L\right)$, with $t$ in $S$. This implies that $F_{0}=D_{s} P^{(L)}$, with $s$ defined by $s\left(L^{\prime}\right)=t\left(L^{\prime}+L\right)$ $\left(L^{\prime} \in V^{*}\right)$. Thus $1.3\left(\mathrm{c}^{\prime}\right)$ implies $1.3\left(\mathrm{c}^{\prime \prime}\right)$.

Since $1.3(\mathrm{c})$ is just the case $L=0$ of $1.3\left(\mathrm{c}^{\prime \prime}\right)$, we have the equivalence of (c), $\left(c^{\prime}\right)$ and $\left(c^{\prime \prime}\right)$ in 1.3.

5. Completion of the proof. Assuming now that 1.3(c) holds we prove that $d_{L}$ is independent of $L$, and is in fact equal to $\operatorname{dim} S / S_{0}$. For this it is enough to show that if $D_{s} P_{L}=0$ with $s$ as in 4.3 , then $s=0$. Assume that $s \neq 0$, that $t$ denotes the sum of the terms of highest degree, say $d$, in the expression for $s$, and that $N$ is the degree of $P$, assumed (see 4.1(a)) to be homogeneous. We have seen (three paragraphs back) that $P_{L}=c P+$ higher terms, with $c \neq 0, c$ in $K$. Since the terms of degree $N-d$ in $D_{s} P_{L}$ are 0 , we get $D_{t} P=0$, then $(t, F)=0$ for all solutions $F$ of $\Sigma_{0}$ by $1.3(c)$, whence $t$ is in $S_{0}$ by 1.2(c). From the way in which $s$ and $t$ have been chosen, this implies $t=0$, a contradition. Thus $d_{L}=\operatorname{dim} S / S_{0}$, a number independent of $L$.

Next assume that 1.3(d) holds. If $L$ is chosen so that $G_{L}$ consists of the identity alone, then $d_{L}=g$ by $1.2(\mathrm{a})$ and $1.2(\mathrm{~b})$. Thus $d_{0}=g$ by $1.3(\mathrm{~d})$, which is $1.3(\mathrm{e})$.

The equivalence of 1.3(e) and 1.3( $\left.\mathrm{e}^{\prime}\right)$ follows from 1.2(c). Assume 1.3( $\left.\mathrm{e}^{\prime}\right)$ holds. Let $s_{1}, s_{2}, \cdots, s_{g}$ be homogeneous elements of $S$ which form a basis for a space complementary to $S_{0}$ in $S$. Every element of $S$ can be written $s=\sum i_{b} s_{b}\left(i_{b} \in I\right)$, and by Galois theory [9,p.156], the expression is unique. Write $p_{a}=\Sigma i_{a b} s_{b}\left(i_{a b}\right.$ homogeneous and in $I$ ), so that $\sum \sum i_{a b} s_{b} i_{a}=0$, whence $\sum i_{a b} i_{a}=0$ for $b=1,2, \cdots, g$. If some $i_{0 b}$ is not in $I_{0}$, it is of degree 0 and we may take it to be 1 . But then the $b$ th equation above yields $i_{0}+\left(i_{1 b} i_{1}+\cdots+i_{r b} i_{r}\right)=0$, contradicting the original assumptions on $i_{0}$. Thus every $i_{0 b}$ is in $I_{0}$ and $p_{0}$ is in $S_{0}$, which is $1.3(\mathrm{f})$.

Now Chevalley [1] has proved that $1.3(\mathrm{f})$ implies $1.3(\mathrm{~g})$, and Shephard and Todd [7] have proved that $1.3(\mathrm{~g})$ implies $1.3(\mathrm{a})$. Thus the cycle is complete, and 1.3 has been established.

6. Proof of 1.4. Assume that $G$ is a reflection group. We have proved in $\S 3$ that $1.3(\mathrm{c})$ is true with $P=\Pi$. If $P^{\prime}$ is another homogeneous polynomial for which 
1.3(c) is true, then $P^{\prime}$ is a derivative of $\Pi$ and of the same degree as $\Pi$, so is a constant multiple of $\Pi$, which proves 1.4(a). At the same time, this shows that the polynomial $P_{0}=P^{(0)}$ of $1.3\left(\mathrm{c}^{\prime}\right)$ is a constant multiple of $\Pi$, whence, by 3.1(a), $\varepsilon=\mathrm{det}$, which is $1.4(\mathrm{~b})$. Now by the equivalence of (a), (c) and (c" $\left.\mathrm{c}^{\prime \prime}\right)$ in $1.3, G_{L}$ is a reflection group. Applying 3.1 to this group, we see that among the nonzero polynomials that transform under $G_{L}$ according to det, the nonzero constant multiples of $\Pi^{(L)}$ are those of minimal degree, which implies 1.4(c). Now if $P_{L}$ is a solution of $\Sigma_{L}$ which transforms under $G$ according to det, then $P_{L}=D_{s} \Pi_{L}$ with $s$ in $S$, by (c), and by averaging over $G$ we may take $s$ in $I$. But then $P_{L}=s(L) \Pi_{L}$ because $\Pi_{L}$ satisfies $\Sigma_{L}$, and this is (d).

As a consequence of $1.4(\mathrm{~d})$, it may be observed that 1.3 and 1.4 are substantially true if the base field $K$ is taken to be the real field, if $L$ is taken in the complexification of $V^{*}$ so that all $i(L)(i \in I)$ are real, and only real solutions of $\Sigma_{L}$ are considered. For then by 1.4(d) both the real and imaginary parts of $\Pi_{L}$ are multiples of $\Pi_{L}$, and whichever of these is nonzero can take the place of $\Pi_{L}$ in the development.

7. Reflection subgroups. It is enough to prove 1.5 in the case that $\operatorname{dim} U=1$ since the general case then follows by induction. Thus, going over to the dual group, we need only prove that if $L$ is in $V^{*}$ then $G_{L}$ is a reflection group. But, as has already been remarked, this is a consequence of the equivalence of (a), (c) and $\left(\mathrm{c}^{\prime \prime}\right)$ in 1.3.

In 1.6 , the subgroup that fixes $v$ is a reflection group by 1.5 , but contains no reflections by assumption, hence consists of the identity, which proves 1.6.

Under the assumptions of 1.7, let $\sigma$ in $G$ permute the $R_{h}$ among themselves, let $k$ be the order of $\sigma$, and let $w=v+\sigma v+\cdots+\sigma^{k-1} v$. Since $R_{h}\left(\sigma^{j} v\right)=\left(\sigma^{-j} R_{h}\right)(v)$ and $\sigma$ permutes the $R_{h}$, the real part of every $R_{h}(w)$ is positive, so that $w$ is not on any reflecting hyperplane for $G$. Since $\sigma w=w$, we conclude that $\sigma$ is the identity, by 1.6 .

8. Difference equations and mean values. For $v$ in $V$, we use $T_{v}$ to denote the translation operator that acts on $F$ in $S^{*}$ via the rule $\left(T_{v} F\right)\left(v^{\prime}\right)=F\left(v^{\prime}+v\right)$, and we call a finite sum $\Sigma c_{v} T_{v}\left(c_{v} \in K\right)$ a difference operator. If $G$ is a finite group of automorphisms of $V$, then in analogy with our previous development we are especially interested in the characteristic functions of those difference operators that are invariant under $G$. This leads us to the following system of difference equations, to be solved for $F$ in $S^{*}$.

$$
g^{-1} \sum_{\sigma \in G} T_{\sigma v} F=C(v) F \quad(v \in V, C(v) \in K) .
$$

We remark that L. Flatto [3] has considered equations such as 8.1 with $C=1$ and $G$ the symmetry group of a tetrahedron or octahedron. Now if $F$ satisfies $\Sigma_{L}$ (see 1.1), then by Taylor's formula $\left(T_{v}=\exp D_{v}\right), F$ satisfies 8.1 with $C$ given by: 


$$
C=g^{-1} \sum_{\sigma \in G} \exp \sigma L
$$

Conversely, assume that the system of equations 8.1 has a nonzero solution $F$. (Here it is enough to assume that $F$ is continuous on some open set in $V$, since then $F$ is infinitely differentiable there (see [5, p. 438]), and this is all we need for the argument that follows. Similarly in our previous development it is enough to assume that the solution $F$ of $\Sigma_{L}$ is a distribution on some open set in $V$ (see 2.1).) Setting $F_{w}(v)=g^{-1} \Sigma\left(T_{\sigma v} F\right)(w)$ for each $w$ in $V$, we see that $\left(D_{i} F_{w}\right)(0)=\left(D_{i} F\right)(w)$ if $i$ is in $I$, whence 8.1 yields $D_{i} F=(i, C) F$. But then (see the discussion before 1.1) we have $(i, C)=i(L)$ for some $L$ in $V^{*}$, and $F$ satisfies the corresponding system $\Sigma_{L}$. Here $\Sigma_{L}$ is uniquely determined by $C$, and by our previous discussion $C$ is given in terms of $L$ by 8.2 . We summarize:

8.3 The system 8.1 has a nonzero solution if and only if $C$ has the form 8.2 for some $L$ in $V^{*}$. The system of difference equations $\Sigma_{L}^{\prime}$ composed of 8.1 and 8.2 is equivalent to the system of differential equations $\Sigma_{L}$ of 1.1 .

From 8.3 we get at once:

8.4 THEOREM. If $\Sigma_{L}$ is replaced by $\Sigma_{L}^{\prime}$ in $1.2,1.3$ and 1.4 , the results there remain true.

Finally, let us remark a characteristic property of the function $C$ of 8.2.

8.5 The function $C$ of 8.2 is a solution of $\Sigma_{L}$, it is invariant under $G$, and it satisfies $C(0)=1$. It is uniquely determined by these properties.

It is clear that $C$ has the stated properties. Assume that $C^{\prime}$ does also. Let $s$ be any element of $S$ and $i$ its average under $G$. Then $\left(s, C^{\prime}-C\right)$ $=\left(i, C^{\prime}-C\right)=\left(D_{i}\left(C^{\prime}-C\right)\right)(0)=i(L)\left(C^{\prime}-C\right)(0)=0$, whence $C^{\prime}-C=0$.

\section{BIBLIOGRAPHY}

1. C. Chevalley, Invariants of finite groups generated by reflections, Amer. J. Math. 77 (1955), 778-782.

2. E. Fiscker, Über algebraische Modulsysteme und lineare homogene partielle Differentialgleichungen mit konstanten Koeffizienten, J. Reine Angew. Math. 140 (1911), 48-81.

3. L. Flatto, Classes of polynomials characterized by a mean value property, Abstract 588-24, Notices Amer. Math. Soc. 9 (1962), 33.

4. Harish-Chandra, Differential operators on a semi-simple Lie algebra, Amer. J. Math. 79 (1957), 87-120.

5. S. Helgason, Differential geometry and symmetric spaces, Academic Press, New York, 1962.

6. N. Jacobson, Lie algebras, Interscience, New York, 1962.

7. G. C. Shephard and J. A. Todd. Finite unitary reflection groups, Canad. J. Math. 6(1954), 274-304.

8. Séminaire "Sophus Lie", Ecole Normale Supérieure, Paris, 1955.

9. B. L. van der Waerden, Modern algebra, Vol. 1, Ungar, New York 1949.

UNIVERSITY OF CALIFORNIA,

Los ANgeles, California 